\title{
Les Diaboliques de Barbey d'Aurevilly : Un éloge de la provocation. Regard sur la nouvelle Le bonheur dans le crime ${ }^{1}$
}

\section{(Les Diaboliques, de Barbey d'Aurevilly: elogio de la provocación. Sobre la novela corta La dicha en el crimen)}

\author{
Verónica Murillo Chinchilla² \\ Universidad Nacional, Costa Rica \\ Universidad de Costa Rica
}

résumé

Cet article analyse la nouvelle Le bonheur dans le crime de l'écrivain français, Barbey d'Aurevilly dans le but de mettre en exergue les trois axes sur lesquels se déplie l'idée esthétique de l'auteur : l'image de la femme, la notion d'amour et l'idéalisation de la transgression. L'intention moraliste à la base du recueil Les Diaboliques (auquel appartient Le Bonheur dans le crime), se transforme en une sorte d'éloge de la beauté du Mal.

\section{resumen}

El presente artículo analiza la novela corta Le bonheur dans le crime ( $\mathrm{La}$ dicha en el crimen) del francés Barbey d'Aurevilly, con el objetivo de destacar los tres ejes en torno a los cuales se despliega la idea estética del autor: la imagen de la mujer, la noción de amor y la idealización de la

1 Recibido: 14 de marzo de 2013; 31 de agosto de 2013.

2 Escuela de Literatura y Ciencias del Lenguaje (UNA) y Escuela de Lenguas Modernas (UCR). Correo electrónico: veronikcr@yahoo.fr

$$
L_{\text {etras }} 54 \text { (2013), ISSN 1409-424X; EISSN 2215-4094 } 91
$$


transgresión. La intención moralista que dio origen a la recopilación de Les Diaboliques (al cual pertenece Le Bonheur dans le crime), se transforma de cierta manera en un elogio a la belleza del Mal.

Mots clé : Littérature française, xix $^{\mathrm{e}}$ siècle, Barbey d'Aurevilly, image de la femme, notion d'amour, idéalisation de la transgression, crime, remords, morale.

Palabras clave: Literatura francesa, siglo xix, Barbey d'Aurevilly, imagen de la mujer, noción de amor, idealización de la transgresión, crimen, remordimiento, moral.

\section{Introduction}

Notre intérêt est de développer dans les pages suivantes une réflexion en guise de panorama de l'idée esthétique proposée par Jules Barbey d'Aurevilly dans Les Diaboliques, recueil de six nouvelles paru en novembre 1874, à Paris, chez l'éditeur Dentu³.

L'ouvrage a été vite jugé comme scandaleux, et son auteur mesuré à partir des portraits qu'il a dressés de ses personnages. Or, nous voudrions regarder de près les fils de la trame d'une de ces nouvelles en particulier : Le bonheur dans le crime dans le but de mettre en exergue ce qui se constitue comme une conception particulière de beauté littéraire.

Trois axes nous semblent s'imposer d'eux-mêmes pour représenter la construction esthétique du récit : l'image féminine ; la notion d'amour et l'idéalisation de la transgression. Tous les trois, même en pouvant être traités isolément, sont complémentaires les uns des autres et servent à soutenir une unité d'ensemble dans le récit, aussi bien que dans le recueil.

\section{Le récit}

Dans la nouvelle de Barbey d'Aurevilly, le narrateur, le docteur Torty, raconte l'histoire d'un couple qui cache un secret atroce

3 Jules Barbey d'Aurevilly, Les Diaboliques. Paris : Editions Le livre de Poche, 1978. 
qui, pourtant, ne nuit pas au bonheur parfait de son amour. C'est l'histoire du comte Serlon de Savigny qui, étant marié à l'aristocrate Delphine de Cantor, fait connaissance de Hauteclaire Stassin, fille de soldat, extrêmement habile pour l'exercice de l'escrime et l'équitation. Tous les deux tombent amoureux malgré l'impossibilité qu'ils ont de mener au bout un projet de vie ensemble.

Un jour, sans raison apparente, Hauteclaire disparaît du village, nul ne connaît son sort pendant des années. C'est seulement le docteur Torty qui parvient à décerner le mystère quand il est appelé chez le Comte pour s'occuper de la comtesse malade et il découvre Hauteclaire habillée comme la femme de chambre.

Curieux de cette découverte, le docteur commence à guetter les protagonistes de l'histoire ; il découvre que Hauteclaire et Serlon mènent une double vie, qu'ils se voient pendant la nuit et qu'ils partagent la passion charnelle et l'amour de l'escrime. Le docteur découvre aussi que la comtesse ne se remettra pas de sa maladie car ce qui se passe en vérité c'est qu'elle meurt victime d'empoisonnement. Plus déconcertant encore c'est que la comtesse est au courant de ce qui lui arrive, mais par une sorte de conscience d'aristocratie, elle refuse de dénoncer son mari et de provoquer le scandale.

Ainsi, l'assassinat s'accomplit dans l'impunité totale, et deux ans après la mort de la comtesse, Serlon de Sévigny épouse Hauteclaire de Stassin. Ils restent un couple parfait dans son bonheur, dans son amour et dans son apparence de jeunesse éternelle. Ils vivent dans leur monde, sans paraître éprouver le moindre remords envers leurs actions passées.

\section{L'image féminine}

Ainsi, nous allons nous occuper de prime abord du traitement narratif octroyé par Barbey d'Aurevilly à l'image de la femme. Dans Le bonheur dans le crime il y a deux personnages féminins qui représentent l'opposition. Il s'agit de Hauteclaire Stassin (la 
fille qui deviendra comtesse) et Delphine de Cantor (victime de l'empoisonnement).

En effet, tout oppose les deux femmes : l'apparence physique (Hauteclaire est grande et forte ; Delphine pâle et frêle) ; le caractère (Hauteclaire est déterminée, Delphine se laisse trâner par la situation). Il suffit de lire attentivement les termes utilisés par l'auteur dans les descriptions pour mieux valoriser ce jeu d'oppositions.

Hauteclaire Stassin, personnage principal du récit, est peinte à plusieurs reprises de façon à montrer au lecteur sa forte personnalité et sa puissance physique. Il s'agit d'une femme élevée par son père dans l'exercice de l'équitation et de l'escrime, le résultat est donc attirant par tout ce qu'il a d'appréciable au regard du lecteur.

Elle était, je dois le dire, très imposante, et elle avait mis tout le monde sur le pied du respect avec elle, n'étant, elle, ni familière, ni abandonnée avec qui que ce fût. Sa physionomie, extrêmement fière, et qui n'avait pas alors cette expression passionnée dont vous venez d'être si frappé, ne trahissait ni chagrin, ni préoccupation ${ }^{4}$.

Ainsi, Hauteclaire est décrite comme ayant des qualités extraordinaires, et surtout, enviables ; elles sont beaucoup plus prisées que les qualités de sa contrepartie : le portrait de Delphine révèle une autre image :

C'était une de ces femmes de vieille race, épuisée, élégante, distinguée, hautaine, et qui, du fond de leur pâleur et de leur maigreur, semblent dire : «Je suis vaincue du temps, comme ma race; je me meurs, mais je vous méprise !» et, le diable m'emporte, tout plébéien que je suis, et quoique ce soit peu philosophique, je ne puis m'empêcher de trouver cela beau ${ }^{5}$.

4 Barbey d'Aurevilly, 95.

5 Barbey d'Aurevilly, 98. 

Ce qui est significatif, c'est qu'à travers les détails physiques sont livrés au lecteur les détails sur le niveau social de chacune des femmes : l'une raffinée et aristocrate, l'autre, d'origine populaire, fille de soldat. Les adjectifs choisis par l'auteur remarquent leurs origines, mais aussi, et surtout, leur décadence.

Le choix des prénoms doit, de même, attirer notre attention. Ce n'est pas gratuit que le prénom de la comtesse rappelle la charge de l'héritage : Delphine évoque immédiatement la notion d'aristocratie et l'importance du Dauphin pour les siens. Hauteclaire, quant à elle, est revêtue par la composition de ce nom étrange, de la prééminence représentée par l'image de la Hauteur de la Lumière (c'est ce qui évoque l'union de ces deux adjectifs, une entité, un nouvel ordre qui passe au-dessus de l'ordre existant).

Dans la thèse doctorale La destruction dans l'œuvre de narrative de Barbey d'Aurevilly, Jésus Sandoval affirme que l'auteur construit ses personnages féminins sur les modèles d'humeur venant du Moyen Age ; il affirme que les femmes peuvent être classifiées selon qu'elles soient lymphatiques ou sanguines. Cette affirmation semble valable particulièrement quand il s'agit de contraster Delphine et Hauteclaire, et la manière dont le tempérant sanguin domine sur le lymphatique.

C'était une femme blanche, molle de tissus, mais dure d'os, au teint de lait dans lequel eût surnagé du son, car les petites taches de rousseur dont il était semé étaient certainement plus foncées que ses cheveux, d'un roux très doux. Quand elle me tendit son bras pâle, veiné comme une nacre bleuâtre, un poignet fin et de race, où le pouls à l'état normal battait languissamment, elle me fit l'effet d'être mise au monde et créée pour être victime... pour être broyée sous les pieds de cette fière Hauteclaire ${ }^{6}$.

6 Barbey d'Aurevilly, 103. 
On peut voir clairement que Delphine, tout en étant d'origine noble, n'a pas d'autre force dans son personnage, elle «se laisse faire », ce qui convient à merveille au caractère de Hauteclaire qui ne recule devant les obstacles. Ainsi, la victime devient proie facile. D'après les affirmations de Sandoval (2008), il s'agit des femmes faibles, trop sensibles et trop attachées à la pudeur, souvent mariées à des hommes qu'elles n'aiment pas ; habituellement elles sont enfermées à la maison et elles n'ont pas de distractions, ce sont des femmes-ornements, qui passent leur vie à l'attente des événements extérieurs.

Elles sont donc, facilement atteintes par la maladie et excessivement fragiles pour la surmonter. Dans le cas de Delphine de Cantor, ce trait est d'autant plus important puisqu'elle «participe » à son meurtre. Ce qui avait été perçu d'abord comme une maladie inexplicable, prend peu à peu sa véritable identité de crime prémédité ; mais Delphine, au lieu de vouloir en fuir, l'accepte comme une façon d'échapper à une existence à laquelle elle ne tient pas vraiment. Cela veut dire qu'à cause de son tempérament lymphatique elle n'affronte pas les obstacles, mais elle les consent, et par là, elle s'autodétruit.

Ces deux femmes, à qui tout oppose, partagent pourtant un trait significatif : l'orgueil. Chez Barbey d'Aurevilly, il n'est pas question de femmes floues du point de vue de leur personnalité, qu'elles soient victimes ou bourreaux. On le voit dans la « conscience de race » de Delphine aussi bien que dans l'effronterie de Hauteclaire. Dans le cas de Delphine, même si la trahison de son mari lui est hideuse, elle ne veut pas que la vérité soit connue, pace qu'elle ne veut pas agir comme son mari, de manière indigne envers leur statut social. Ce sont ses mots qui expliquent ces raisonnements :

Il aime cette scélérate, cette exécrable fille qui m'a tuée. Il est plus coupable qu'elle, puisqu'il l'aime et qu'il m'a trahie pour elle. [...] Je ne veux pas, quand je serai morte, que le comte de Savigny passe pour l'assassin de sa femme. Je ne veux pas qu'on le traîne en cour d'assises, qu'on l'accuse de complicité avec une servante adultère et 
empoisonneuse ! Je ne veux pas que cette tache reste sur ce nom de Savigny, que j'ai porté7.

La supériorité vantée par Delphine est la seule qu'elle peut faire valoir contre Hauteclaire, sa conscience de statut social est si forte, que l'envie de ne pas nuire à sa noblesse l'emporte, même sur le désir de se venger de la trahison de son mari en révélant le lien scandaleux, et par ce biais, sauver sa vie.

En ce qui concerne Hauteclaire, tous les détails fournis au lecteur sur l'apparence ou la personnalité de la femme renforcent cette conception de supériorité envers les autres. L'héroïne d'Aurevilly a été parée de qualités qui la rendent extraordinaire, aussi bien du point de vue physique que du point de vue moral, et ce choix narratif va lui donner un statut au-delà des notions du Bien et du Mal.

Cette femme, en effet, prenait encore plus le regard que l'homme qui l'accompagnait, et elle le captivait plus longtemps. Elle était grande comme lui. Sa tête atteignait presque à la sienne. Et, comme elle était aussi tout en noir, elle faisait penser à la grande Isis noire du Musée Egyptien, par l'ampleur de ses formes, la fierté mystérieuse et la force. Chose étrange ! dans le rapprochement de ce beau couple, c'était la femme qui avait les muscles, et l'homme qui avait les nerfs... Je ne la voyais alors que de profil ; mais ; le profil, c'est l'écueil de la beauté ou son attestation la plus éclatante. Jamais, je crois, je n'en avais vu de plus pur et de plus altier ${ }^{8}$.

De cette manière se constitue l'héroïne par excellence chez Barbey d'Aurevilly, une femme qui sait se montrer froide, hautaine, silencieuse et pleine d'orgueil, ce sont là les traits diaboliques qui ont tant touché le public au moment de leur parution : des personnages sans scrupules, exempts du repentir et déterminés jusqu'au bout.

7 Barbey d’Aurevilly, 117.

8 Barbey d'Aurevilly, 86. 
En plus, il s'agit de femmes hors du commun, par les traits de leur caractère, leurs caractéristiques physiques et leurs constructions émotionnelles, elles acquièrent une dimension mythique au-dessus de l'humain. Le choix nous semble important à remarquer puisqu'il s'agit d'un ouvrage du XIX ${ }^{\mathrm{e}}$ siècle, où il était plutôt question dans la société française d'un retour à la morale conservatrice.

Quels motifs poussent donc Barbey d'Aurevilly à la construction des héroïnes qui vont à l'encontre du « politiquement correct »? S'agit-il d'un défi ou d'une critique ? Ou nous sommes devant une manifestation littéraire qui ne correspond pas tout-à-fait à son époque ? Nous reviendrons sur ces considérations.

\section{La notion de l'amour}

Dans Le bonheur dans le crime, l'amour doit être assumé comme une passion, dans le sens le plus fort du mot. Il s'agit d'un sentiment qui ne connaît ni raison, ni assouvissement, ni limites : c'est un but qui justifie TOUS les moyens. C'est par cette puissance qu'il devient hors du contrôle humain et donc : diabolique.

Le sentiment de l'amour se définit alors en relation avec ceux d'érotisme, hardiesse, provocation, dissimulation et mystère. En effet, l'aspect essentiel c'est qu'il s'agit d'un amour adultère qui s'impose et dure dans le temps. La naissance et consolidation de l'amour entre Hauteclaire et Serlon de Savigny sont marquées par la présence du danger et la défiance aux risques, il s'agit d'une provocation envers l'entourage, mais aussi envers le lecteur : «Il est des passions que l'imprudence allume, et qui, sans le danger qu'elles provoquent, n'existeraient pas. $»^{9}$

De cette façon les deux amants poussent leur hardiesse jusqu'au point de faire entrer Hauteclaire au service de la comtesse, pour pouvoir partager des moments d'intimité pendant la nuit. Le

9 Barbey d'Aurevilly, 101. 
docteur Torty, nous en rapporte témoignage : « J'avais arrêté mon cheval sur le bord du bois, écoutant leur engagement qui paraissait très vif, intéressé par cet assaut d'armes entre amants qui s'étaient aimés les armes à la main et qui continuaient de s'aimer ainsi $\gg{ }^{10}$.

Le consentement du comte de Savigny pour installer sa concubine à la maison, favorise toute sorte d'échanges érotiques sous les yeux de la comtesse. Au début, Serlon se sent mal à l'aise, mais pendant la nuit ils ont une existence parallèle, pleine d'érotisme et de provocation. Le couple, en tant qu'exceptionnel, vit son amour d'une manière aussi exceptionnelle : ils se rencontrent loin des pièces principales de la maison, et ils mélangent amour et escrime, de manière à ce qu'ils se retrouvent dans le danger et dans le plaisir.

Une nuit, le docteur les découvre au milieu de ces scènes, il décrit les détails :

Serlon et Hauteclaire vinrent s'accouder sur la rampe en fer du balcon [...]. Hauteclaire était vêtue, si cela s'appelle vêtue, comme je l'avais vue tant de fois, donnant ses leçons à $\mathrm{V}$... [...], lacée dans ce gilet d'armes de peau de chamois qui lui faisait comme une cuirasse [...] dans ces vêtements serrés qui ressemblaient à une nudité.

[...] ils formèrent à eux deux ce fameux et voluptueux groupe de Canova qui est dans toutes les mémoires, et ils restèrent ainsi sculptés bouche à bouche le temps, ma foi, de boire, sans s'interrompre et sans se reprendre, au moins une bouteille de baisers! Cela dura bien soixante pulsations comptées à ce pouls qui allait plus vite qu'à présent, et que le spectacle fit aller plus vite encore ${ }^{11}$.

La description suggère un échange sexuel explicite, mais elle est chargée d'érotisme par les détails du décor : la nuit, la lune, le silence... et les armes qui les unissent tous deux, comme ayant accès à un monde exclusif. C'est d'ailleurs la conclusion obligatoire dans l'ensemble de la nouvelle.

10 Barbey d'Aurevilly, 107.

13 BRarbeydd Àtremily,y 85 !1. 
Cette intimité repose aussi bien sur d'autres risques, comme l'affirme Torty : «En voyant ces caresses et cette intimité qui me révélaient tout, j'en tirais, en médecin, les conséquences ${ }^{12}$. Il est évident, par ces mots que le docteur attend l'apparition d'une grossesse qui, pourtant, n'est jamais arrivée.

Le dénouement est prévisible : la comtesse devient un obstacle à éliminer, et l'empoisonnement, la meilleure option. De cette manière, l'amour s'appuie aussi sur le machiavélisme. On l'a déjà mentionné : le but justifie les moyens, la stratégie se construit impitoyablement : la complicité en cachette, le meurtre, la cohabitation et le mariage : un amour qui résiste toutes les épreuves : le temps, le vieillissement, les conventions sociales...la morale.

La preuve en est fournie par le docteur Torty dans deux détails qui rapportent au passage du temps et à la perpétuation de leur passion.

C'étaient un homme et une femme, tous deux de haute taille, et qui, dès le premier regard que je leur jetai, me firent l'effet d'appartenir aux rangs élevés du monde parisien. Ils n'étaient jeunes ni l'un ni l'autre, mais néanmoins parfaitement beaux. L'homme devait s'en aller vers quarante-sept ans et davantage, et la femme vers quarante et plus... Ils avaient donc, comme disent les marins revenus de la Terre de Feu, passé la ligne, la ligne fatale, plus formidable que celle de l'équateur, qu'une fois passée on ne repasse plus sur les mers de la vie ! Mais ils paraissaient peu se soucier de cette circonstance. Ils n'avaient au front, ni nulle part, de mélancolie...

Ils étaient superbes, en s'éloignant ainsi, sous les rayons du soleil de midi, dans la majesté de leur entrelacement, ces deux êtres... ${ }^{13}$.

Il est évident qu'ils ont résisté le passage du temps, mais non seulement en ce qui concerne leurs sentiments, leurs apparences physiques semblent le défier aussi. À cela, il faut ajouter que dans le 

cours du récit, le docteur mentionne l'inexistence d'enfants dans ce couple, ce détail devient autre des piliers de leur passion :

-Vous n'êtes donc pas triste de n'avoir pas d'enfant, madame la comtesse?

-Je n'en veux pas ! fit-elle impérieusement. J'aimerais moins Serlon. -Les enfants, ajouta-t-elle avec une espèce de mépris, sont bons pour les femmes malheureuses! ${ }^{14}$

C'est dans ce sens, qu'il est possible d'affirmer que chez Barbey d'Aurevilly la notion d'amour renvoie à celle de passion, et par là, elle fait référence à ce qu'il y a de plus instinctif dans l'être humain. La phrase n'est pas exemptée de la référence à la recherche hédoniste, à l'égoïsme partagé de cet amour réciproque et exclusif. La présence d'un enfant viendrait briser l'unité établie entre Serlon et Hauteclaire, elle est, clairement, indésirable.

La combinaison entre amour et danger fournit aux amants une complicité qu'ils ne pourraient pas atteindre par d'autres moyens, ils procurent la complaisance devant le risque et le désir de choquer, leur mariage est provocation et défi en même temps, c'est une effronterie contre tout ce qui représente la vie dans un petit village.

Ils refusent même de cacher leur passion et ne veulent pas abandonner le scénario de crime, leur bonheur est pour eux légitime, et ils ne cherchent pas à le cacher, quoiqu'en disent les autres. La société, de sa part, les abandonne, mais ils la défient, parce qu'ils semblent encore plus heureux dans leur isolement.

Mais pourquoi ne s'en vont-ils pas du pays? Le comte est riche. Il peut vivre grandement partout. Pourquoi ne pas filer avec cette belle diablesse? [...] Il y avait là un dessous que je ne comprenais pas [...]. Hauteclaire, que je croyais l'homme des deux dans leurs rapports d'amants, voulait-elle rester dans ce château où on l'avait vue servante

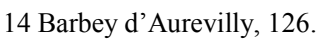


et où l'on devait la voir maîtresse, et en restant, si on l'apprenait et si cela faisait un scandale, préparer l'opinion à un autre scandale, bien plus épouvantable, son mariage avec le comte de Savigny? ${ }^{15}$

On voit bien que la notion d'amour relève aussi bien de la volupté du secret, de la simulation, de la rébellion contre la rigidité des normes sociales. L'union des deux amants est un défi contre la morale et les conventions qui s'opposent au mariage entre nobles et plébéiens. Cette relation ne peut être valorisée dans sa juste mesure qu'à partir du contexte social dans lequel elle s'insère, puisque le choix du comte scandalise son entourage mais il devient aussi la base de son bonheur et l'absence de remords.

A l'encontre des valeurs traditionnelles, leur bonheur dans le crime met en question les bases de la morale chrétienne fondées sur les notions de péché et de châtiment. Ces amants cherchent le scandale par leur libre arbitre, ils l'atteignent par la permanence et la durabilité de leur amour. Le crime apparaît finalement comme un des composants dudit amour, c'est la conclusion d'une rivalité entre « races », Hauteclaire, défie et vainc ce que la comtesse voulait protéger : la «bonne société »: la perpétuation de cet amour devient alors directement proportionnelle à la violence du défi lancé contre l'opinion des gens respectables.

Mais le mariage, le mariage effrontément accompli au nez de Dieu et des hommes, mais ce défi jeté à l'opinion de toute une contrée outragée dans ses sentiments et dans ses mœurs. [...] Les femmes du pays, qui, comme autrefois, passent en voiture, la dévisagent plus encore peut-être que quand elle était la grande et mystérieuse jeune fille au voile bleu sombre, et qu'on ne voyait pas. Maintenant elle lève son voile et leur montre hardiment le visage de servante qui a su se faire épouser ${ }^{16}$.

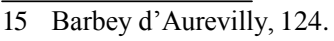

16 Barbey d'Aurevilly, 124. 
En essence, il s'agit d'un amour qui se suffit à lui-même : le couple refuse la procréation, la femme se soumet à l'objet de son amour et va jusqu'au bout pour assurer sa consommation. Entre eux, la distinction de sexes disparaît, l'homme laisse paraître des traits féminins, en même temps que la femme fait preuve d'une évidente masculinité. Ils fondent l'un dans l'autre et l'intensité de leur passion les fait se passer de leur individualité : ils vont partout ensemble, ils chevauchent et parcourent les collines, ils ont des passionnantes parties d'escrime de même qu'ils partagent les moments d'oisiveté à la maison. Le docteur le résume ainsi : « cet idéal réalisé par eux, et que je croyais impossible, dégoûté des meilleurs mariages que j'aie connus, et que le monde appelle charmants. Je les ai trouvés si inférieurs au leur, si décolorés et si froids! ${ }^{17}$

\section{L'idéalisation de la transgression}

Voici le dernier point sur lequel nous voudrions porter notre attention. Il a été déjà mentionné à maintes reprises le caractère particulier de ce récit «aurévilien »; non seulement par le suggestif d'un titre comme Les Diaboliques, mais aussi par le choix narratif d'une mise en scène exquise dans les détails, dans les situations narratives, dans le délicat équilibre entre sensualité et horreur.

Horreur dans le sens que les thèmes abordés visent à provoquer le rejet chez le lecteur, une espèce de condamnation morale, il est vrai ; mais il est aussi vrai que la lecture agit comme un acte de séduction, et que le lecteur est facilement dupe d'une sorte de fascination morbide.

Premièrement, nous voulons attirer l'attention sur le fait que Les Diaboliques est conçu par son auteur comme un ouvrage moralisant visant à mettre en garde contre les astuces féminines. Les donnés biographiques sur Barbey d'Aurevilly témoignent de cet intérêt

17 Barbey d'Aurevilly, 125. 
ainsi que de ses relations harmonieuses avec la religion chrétienne. En fait, rien d'étonnant pour une époque où la société veut un retour aux bonnes mœurs et l'élimination de la déchéance vécue sous l'Ancien Régime.

Cependant, une fois mis l'accent sur cet aspect particulier, nous voulons nous en servir pour remarquer l'autre caractéristique que nous avons définie comme essentielle au récit : la mise en scène de la transgression finit par fasciner le lecteur.

C'est-à-dire que, pour ce qui concerne notre perception, tout en voulant horrifier contre la bassesse morale et la perversité, Barbey d'Aurevilly est parvenu à créer un univers séduisant, où le crime perd sa connotation condamnable qui va se dissoudre dans la peinture d'une histoire extraordinaire entre deux êtres aussi extraordinaires ; la transgression est ainsi idéalisée dans un sens dépourvu de morale.

Cet effet ne peut s'atteindre que par la conjonction de tous les éléments narratifs, ceux qui portent sur la forme à côté de ceux qui portent sur le fond. C'est l'équilibre entre la description d'une femme charmante, admirable, amoureuse et le témoignage d'un amour parfait et inépuisable. Même pas le sordide du meurtre ne peut ternir l'éclat de leur bonheur.

Mais la transgression se manifeste sur plusieurs domaines, qui vont au-delà de l'histoire mise en scène. D'abord il s'agit, (on l'a déjà mentionné) de la transgression des classes sociales : une plébéienne qui se marie à un noble ; ensuite, il y a très présente la transgression de rôles, dans ce cas on peut parler d'une certaine androgynie.

En effet, Hauteclaire Stassin met en question le concept traditionnel de féminité, elle ne grandit pas à côté d'une figure maternelle, et son père lui apprend des disciplines «masculines » comme l'escrime et l'équitation, ce qui lui donne une apparence physique qui diffère de celle féminine : «[...] c'était un marmot solide que cette fillette, avec des attaches et des articulations d'acier fin, il la développa d'une si étrange manière, qu'à dix ans elle semblait en 
avoir déjà quinze, et qu'elle faisait admirablement sa partie avec son père et les plus forts tireurs de la ville de $\mathrm{V}$... ${ }^{18}$.

Par contre, il existe une féminisation du rôle masculin chez le comte, avec : " son air efféminé et hautain ». Ainsi, les rôles s'inversent : "C'était la femme qui avait les muscles et l'homme qui avait les nerfs ${ }^{19}$. L'image est renforcée par l'affrontement qu'ils ont, où il n'arrive pas à toucher Hauteclaire, qui est beaucoup plus habile.

Il existe aussi la transgression des conventions sociales en ce qui concerne les bonnes mœurs : Hauteclaire met le voile pour ne pas être vue des gens du village, mais le voile la rend beaucoup plus sensuelle :

Mlle Stassin apparaissait, trottant ou galopant dans la perspective d'une route [...]. Seulement [...] elles [les jeunes filles du pays] n'avaient jamais bien vu que la tournure de cette fille, faite pour l'amazone [...] dont le visage était toujours plus ou moins caché dans un voile bleu gros trop épais [...]. Elle se montrait très rarement dans la rue, - et les femmes comme il faut ne pouvaient la voir que là, ou encore le dimanche à la messe; mais, le dimanche à la messe, comme dans la rue, elle était presque aussi masquée que dans la salle de son père, la dentelle de son voile noir étant encore plus sombre et plus serrée que les mailles de son masque de fer ${ }^{20}$.

C'est un voile noir, épais, hermétique, il suggère le mystère de l'intrigue. Ce voile favorise une certaine ambigüité : on présume la femme innocente et pure ; pourtant la valeur du voile c'est de lui permettre la dissimulation, le secret, la provocation.

Un autre aspect où se présente une transgression sociale c'est le rôle du docteur Torty. Il s'agit d'un personnage ambivalent : collaborateur et dénonciateur, il est confident et témoin privilégié, mais aussi complice du couple. En plus, il joue le rôle de celui qui accède aux

18 Barbey d'Aurevilly, 91.

19 Barbey d'Aurevilly, 86.

20 Barbey d'Aurevilly, 93. 
secrets sans attendre qu'ils lui soient confiés, il transgresse aussi ses devoirs professionnels pour satisfaire sa curiosité, et alors il devient une sorte de voyeur, tout son récit naît de cette curiosité qui le travaille et le fait épier ses personnages, même au milieu de l'obscurité:

Ah! les plaisirs de l'observateur! ces plaisirs impersonnels et solitaires de l'observateur, que j'ai toujours mis au-dessus de tous les autres, j'allais pouvoir me les donner en plein, dans ce coin de campagne, en ce vieux château isolé, où, comme médecin, je pouvais venir quand il me plairait... Serlon et Hauteclaire vinrent s'accouder sur la rampe en fer du balcon [...]. Hauteclaire était vêtue, si cela s'appelle vêtue, comme je l'avais vue tant de fois, donnant ses leçons à V... [...], lacée dans ce gilet d'armes de peau de chamois qui lui faisait comme une cuirasse [...] dans ces vêtements serrés qui ressemblaient à une nudité ${ }^{21}$.

En synthèse, il est possible d'affirmer, que toutes ces conduites de transgression n'ont pas le caractère négatif auquel elles sont associées dans d'autres situations et que dans Le bonheur dans le crime elles revêtent plutôt une apparence séduisante difficile à ternir ou a transformer en désir moralisant.

\section{Conclusion}

Nous voudrions conclure notre réflexion autour de ce récit de Barbey d'Aurevilly en énonçant quelques aspects importants sur l'image féminine, la valeur des sentiments et les implications de la transgression abordée dans un ouvrage du xix ${ }^{\mathrm{e}}$ siècle, vus à la lumière de ce $\mathrm{xxi}^{\mathrm{e}}$ siècle.

D'abord, il nous semble que le récit en question met en scène une narrative d'avant-garde pour son époque, aussi bien dans les situations narrées que dans les personnages créés. Cela expliquerait

$21 \quad$ Barbey d’Aurevilly, 98. 
suffisamment la critique suscitée contre Les Diaboliques et l'assimilation de l'auteur à ses personnages : ce n'est autre chose que la peur de l'inconnu, des personnages qui n'entraient pas dans les valeurs canoniques de l'époque. Dans ce sens, autant dire de la critique contre Les Liaisons dangereuses un siècle auparavant ; dans la même lignée que la réception que Madame Bovary avait eu à peine quelques décennies avant.

Ensuite, même si l'amour est le thème évident dans le récit, il l'est à côté de la mort, et il ne s'agit pas d'une mort quelconque. Le meurtre par empoisonnement rappelle la présence du Mal, de l'Enfer placé sous l'âme, la famille, la société ; c'est le Mal qui se sert de la dissimulation et du secret, qui résiste toutes les impositions morales de son époque, et aux règles littéraires pour montrer au lecteur que sous les surfaces des apparences sociales, il existe toujours des ténèbres. Il s'agit du déploiement de forces liées à l'instinct, le sexe, la force et le pouvoir. Et ici, la question naît tout naturellement : ne s'agit-il pas d'une dénonce contre certains groupes ? Barbey d'Aurevilly deviendrait-il alors une espèce de critique de sa société ?

Si ces nouvelles sont Les Diaboliques, c'est parce qu'elles transgressent des silences accordés pour le «bien commun » et le satanisme qu'y apparaît est essentiellement moral et social, il existe au cœur humain, les personnages sont des êtres en chair et os, guidés par des passions terrestres et leur propre perversité. Dans Le bonheur dans le crime, l'auteur met en évidence que parfois dans la vie le Mal s'impose au Bien, sans remords.

De cette manière, l'univers narratif de Barbey d'Aurevilly propose tout ce qui est contraire à un monde régi par les commandements de l'Église : il nous offre les charmes de l'adultère, des maris qui participent au meurtre de leurs femmes, des femmes sans scrupules et sans faiblesses appuyées sur le «moralement correct». Pour l'auteur, l'essentiel est l'intensité des passions, même si celles-ci conduisent à l'Enfer. Dans ces conditions, le titre va de soi, les passions et les jouissances des personnages sont toujours diaboliques. 
Ainsi, la lecture de Barbey d'Aurevilly éveille deux sentiments contradictoires mais complémentaires : l'horreur et l'admiration. L'imagination du romancier fournit des images effrayantes qui ne cessent pas d'attirer parce qu'elles répondent à certaines passions morbides et malsaines faisant partie de la condition humaine et plus rassurantes pour le lecteur lorsqu'elles font partie d'une intrigue littéraire et non des faits de la vie réelle. 